\title{
Therapeutic Potential of Social Networking Sites for Caregivers
}

\author{
Trey Asbury, Nancy L. Myers \\ Texas Woman's University, Denton, USA
}

\begin{abstract}
SNS (social networking sites) such as Facebook and Twitter are widely used as a source of news, marketing, and entertainment. These online platforms also allow individuals to connect and maintain social ties with others. Researchers have recently begun investigating psychological, emotional, and behavioral correlates of SNS usage. The current study examines the potential benefits of SNS usage for persons serving in a caregiver role. While no differences were observed for perceived social support, SNS usage was negatively related to anxiety and depression levels for caregivers who utilize these platforms frequently.
\end{abstract}

Keywords: SNS (social networking sites), Facebook, caregivers, anxiety, depression

\section{Social Networking Impact on Personal Happiness}

SNS (social networking sites) have been accessible for almost two decades and have changed the way individuals communicate. SNS are generally described as a more dynamic Web. 2.0 platform on the internet that allows users to interact and collaborate (Kramer \& Winter, 2005). Facebook, Twitter, Instagram, and LinkedIn are among the most popular social networking sites (ebizmba.com, December 2013). Geographic restrictions and busy schedules that often impede human interaction make SNS an attractive form of "asynchronous" communication. While motivation to use SNS varies, potential outcomes such as links to well-being have been increasingly under investigation (Asbury \& Hall, 2013).

Subjective well-being is defined by G. Lee, J. Lee, and Kwon (2011, p. 211) as, "a person's cognitive and affective evaluations of his or her life". Lee et al. (2011) consider the lack of social networking cues, or etiquette, a largely unexamined new territory. As a result, individuals have a tendency to show less self-censorship while engaged with SNS as opposed to face-to-face communication. The resulting, less-restrained online environment may provide a catalyst for increasing well-being via added self-disclosure.

Research conducted by Kim and Roselyn Lee (2011) examined the most popular SNS, Facebook, and correlated to psychological well-being for the users. Findings revealed that self-presentation to Facebook friends did contribute to the user's overall sense of well-being, happiness, and social support. Also, Asbury and Hall (2013) found that highly engaged users of Facebook were more likely to report high levels of social support. Similar findings include relationships to high self-esteem, positive affect, and social connectedness (Gonzales \& Hancock, 2011; Morris \& Aguilera, 2012).

The current study examined SNS participation as a potential mechanism of well-being for users engaged in a caregiver role. Historically, multiple self-help resources and support groups have been available to those who provide chronic care for someone with physical or mental disabilities. Researches from the 1980s

Trey Asbury, Ph.D., assistant professor, Department of Psychology and Philosophy, Texas Woman's University. Nancy L. Myers, B.A., Department of Psychology and Philosophy, Texas Woman's University. 
consistently found positive relationships between support groups, mental health, and well-being. Many of these findings relate to the efficacy for coping with stress by sharing and learning (Medvene \& Krauss, 1989; Potasznik \& Nelson, 1984). Powell (1987) summarized the general finding that supports group participants enjoy a sense of catharsis when allowed to share experiences with others who have similar stories.

There are numerous obstacles that prevent the potential benefits of traditional support groups. Limited resources, for example, may exist for those who live in rural environments (Perron, 2002). Furthermore, the demanding nature of the caregiver role may simply prevent attendance to available meetings. Certain disabilities inherently lead to restrictions on time, finances (some support groups charge a fee), and altered sleep and work schedules (Perron, 2002). However, social networking sites are immune to such limitations and provide an attractive alternative for those who are chronically engaged in a caregiver role.

As a result, our study tested the following hypotheses:

H1: Degree of participation with SNS will be related to overall well-being. Caregivers who utilize SNS frequently will report higher life satisfaction than those who seldom utilize SNS.

H2: Degree of participation with SNS will be related to depression. Caregivers who utilize SNS frequently will report less depression compared to those who seldom utilize SNS.

H3: Degree of participation with SNS will be related to anxiety. Caregivers who utilize SNS frequently will report less anxiety compared to those who seldom utilize SNS.

\section{Method}

\section{Participants}

Fifty-nine adults ( 42 women, 17 men, $M$-age $=26.2$ years) were recruited via email listserv at a state university. Snowball sampling procedures encouraged respondents to recruit others via SNS such as Facebook. Qualifications to participate included: (1) 18 years of age or older; (2) account holder and user of SNS (examples of Facebook and Twitter provided); and (3) current status as a primary caregiver to someone with special needs. Prior to recruitment, the study was approved by the university's Institutional Review Board and none of the participants were compensated.

\section{Materials and Procedure}

The recruitment email invited qualified participants to complete an online survey via Psychdata. The survey included a brief demographic section, followed by items assessing social support, depression, and anxiety. Seventy-eight percent of the participants identified as the caregiver of a parent with special needs, $13 \%$ identified as the caregiver of a child with special needs, $7 \%$ identified as the caregiver of both a parent and a child with special needs, and $2 \%$ identified as other. Social support items were from MSPSS (the Multidimensional Scale of Perceived Social Support) (G. Zimet, Dahlem, S. G. Zimet, \& Farley, 1988). The social support scale consists of a 12-item questionnaire based on a 7-point Likert scale. The MSPSS assesses social support from both friends and family. The MSPSS has sound psychometric properties with measures of internal consistency ranging from 0.85 to 0.91 (Zimet, Powell, Farley, Werkman, \& Berkoff, 1990). Depression (14 items) and anxiety (14 items) were assessed from the Depression and Anxiety Stress Scale (S. H. Lovibond \& P. F. Lovibond, 1993), which has demonstrated high internal consistency with Cronbach's alphas of 0.94 and 0.88 for those constructs (Nieuwenhuijsen, de Boer, Berbeek, Blonk, \& van Dijk, 2003). 


\section{Results}

Separate independent sample $t$-tests were performed to examine differences in social support, anxiety, and depression based on frequency of SNS participation. See Table 1 for descriptive data pertaining to each construct, participants were grouped as high versus low frequency of SNS usage based on a median split (median $=3$ hours per week). The median split technique is a commonly used procedure in quasi-experimental designs and poses minimal threats to Type I error rates (Baumester, 1990). A Bonferonni correction was used to protect against the increased risk of Type I error when performing multiple tests. In addition, analysis of covariance procedures examined the potential moderating effects of sex and age of participant.

Table 1

Summary of Descriptives (Social Support, Anxiety, and Depression)

\begin{tabular}{lllll}
\hline Constructs & Min/Max & $\underline{\text { High } f \text { SNS }}$ & Min/Max & $\underline{\text { Low } f \text { SNS }}$ \\
\hline Social support & $30 / 62$ & Mean (Std. Error) & & Mean (Std. Error) \\
Anxiety & $19 / 37$ & $22.4^{*}(0.46)$ & $32 / 58$ & $49.7(0.40)$ \\
Depression & $13 / 35$ & $17.8^{*}(0.33)$ & $21 / 40$ & $31.6^{*}(0.91)$ \\
\hline
\end{tabular}

Note. ${ }^{*} p<0.01$.

\section{Social Support}

The first hypothesis was not supported as mean comparisons of the overall social support score did not differ between high $(M=50.6)$ and low frequency users $(M=49.7)$ of SNS. The null hypothesis was also supported when controlling for sex and age of the participant.

\section{Anxiety \& Depression}

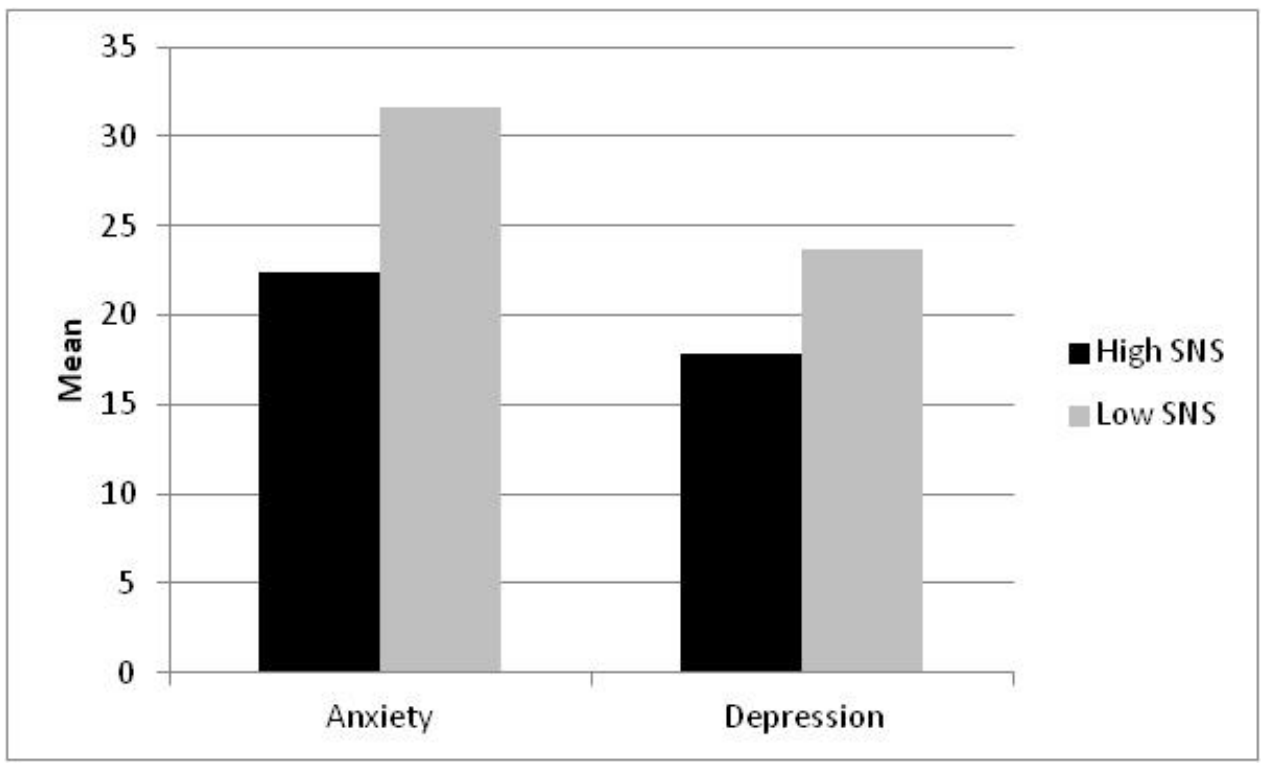

Figure 1. High vs. Low Users of SNS.

Levels of anxiety was significantly higher for low frequency users of SNS $(M=31.6$, S.D. $=3.2)$ compared to high users of SNS $(M=22.4, S . D .=4.8)$. Independent sample $t$-test supported $\mathrm{H} 2, t(58)=5.70, p$ $<0.01$. Difference between groups remained after controlling for sex and age. Support for H3 was indicated as 
low frequency users of SNS reported higher levels of depression $(M=23.7$, S.D. $=2.5)$ compared to high frequency users of SNS $(M=17.8, S . D .=3.1)$. Differences between groups remained after controlling for sex and age; $t(58)=4.31, p<0.01$ (See Figure 1).

\section{Discussion}

There are a relatively large number of self-help and support groups available to caregivers residing in industrialized urban areas. While these resources may provide effective therapeutic intervention, multiple barriers are common for many who simply do not have the time or means to attend. The rising popularity of online support mechanisms such as SNS addresses many of these barriers. Asynchronous communication has also become more popular than synchronous communication as busy individuals can utilize SNS, email, and online bulletin boards at their own convenience (Perron, 2002).

While our findings related to anxiety and depression supported our hypotheses, it remains unclear why there were no differences in our groups related to social support. Ceiling and floor effects were ruled out as an explanation as average scores were just higher than the middle range of the MSPSS. Another possibility for this finding may have been explained by the nature of the caregiver role and items on the scale linked to parental relationships. Since the MSPSS examines social support from friends and family, parental items on the scale may have been influenced by the majority of caregivers who were taking care of parents with special needs. As a result, a follow-up analysis excluded the social support items related to parents, but again, no significant differences were discovered. Further investigation is warranted and the inclusion of multiple scales of measurement for social support is recommended.

There are several shortcomings related to our sample that warrant address. One weakness of the current study is the failure to identify a control group of participants who do not use SNS as the snowball sampling method actually utilized SNS for recruitment. As a result, comparisons between caregivers who are users and non-users of SNS should be investigated. While nearly $60 \%$ of persons worldwide utilize some type of social networking site (statisticsbrain.com, 2014), future studies should seek creative ways to recruit persons who do not use SNS. In addition, we did not collect data about the severity of special needs faced by caregivers. While self-report ratings are objective, disparity in the range of special needs should have been assessed.

While few studies have addressed the therapeutic potential of SNS, research based on internet usage suggests that such communities may actually be more beneficial in terms of emotional support and self-disclosure than the face-to-face counterparts (Salem, Bogar, \& Reid, 1997). Current findings add to the existing literature in this area as lower levels of anxiety and depression was reported by caregivers who frequently use SNS. As the popularity of social networking sites continues to rise, we are encouraged by the potential therapeutic benefits suggested by our findings.

\section{References}

Asbury, E. T., \& Hall, S. (2013). Facebook and other social technologies as a means of social support. Psi Chi Journal of Psychological Research, 18(3), 124-129.

Baumeister, R. (1990). Item variances and median splits: Some encouraging and reassuring findings. Journal of Personality, 58(3), 589-594. doi: 10.1111/j.1467-6494.1990.tb00244.x

Ellison, N., Steinfield, C., \& Lampe, C. (2007). The benefits of Facebook “friends": Social capital and college students' use of online social network sites. Journal of Computer Mediated Communication, 12, 1143-1168.

Gonzales, A. L., \& Hancock, J. T. (2011). Mirror, mirror on my Facebook Wall: Effects of exposure to facebook on self-esteem. Cyberpsychology, Behavior \& Social Networking, 14(1/2), 79-83. 
Lee, G., Lee, J., \& Kwon, S. (2011). Use of social-networking sites and subjective well-being: A study in South Korea. Cyberpsychology, Behavior, and Social Networking 14.3 (2011): 151-155.

Kim, J., \& Roselyn Lee, J. (2011). The Facebook paths to happiness: Effects of the number of Facebook friends and self-presentation on subjective well-being. Cyberpsychology Behavior, and Social Networking, 14(6), 359-364. doi:10.1089/cyber.2010.0374

Ko, H., \& Kuo, F. (2009). Can blogging enhance subjective well-being through self-disclosure?. Cyberpsychology \& Behavior, 12(1), 75-79. doi: 10.1089/cpb.2008.016

Kramer, N. C., \& Winter, S. (2008). Impression management 2.0: The relationship of self-esteem, extraversion, self-efficacy, and self-presentation within social networking sites. Journal of Media Psychology: Theories, Methods, and Applications, 20(3), 106-116. doi: 10.1027/1864-1105.20.3.106

Lovibond, S. H., \& Lovibond, P. F. (1993). Manual for the Depression Anxiety Stress Scales (DASS). University of New South Wales.

Medvene, L. J., \& David, H. K. (1989). Causal attributions and parent-child relationships in a self-help group for families of the mentally ill. Journal of Applied Social Psychology, 19(17), 1413-1430.

Morris, M. E., \& Adrian, A. (2012). Mobile, social, and wearable computing and the evolution of psychological practice. Professional Psychology: Research and Practice, 43(6), 622.

Nieuwenhuijsen, K., de Boer, A., Berbeek, J., Blonk, R., \& van Kijk, F. (2003). The Depression Anxiety Stress Scales: Detecting anxiety disorder and depression in employees absent from work because of mental health problems. Occupational Environmental Medicine, 60(1), 77-82.

Perron, B. (2002). Online support for caregivers of people with a mental illness. Psychiatric Rehabilitation Journal, 26(1), 70-77. doi: $10.2975 / 26.2002 .70 .77$

Potasznik, H., \& Geoffrey, N. (1984). Stress and social support: The burden experienced by the family of a mentally ill person. American Journal of Community Psychology, 12(5), 589-607.

Powell, D. R. (1987). A neighborhood approach to parent support groups. Journal of Community Psychology, 15(1), 51-62.

Salem, D. A., Bogar, G. A., \& Reid, C. (1997). Mutual help goes on-line. The Journal of Community Psychology, 25(2), $189-207$.

Social Networking Statistics. (2014). Retrieved from http://www.statisticbrain.com/social-networking-statistics/

Utz, S., \& Beukeboom, C. J. (2011). The role of social network sites in romantic relationships: Effects on jealousy and relationship happiness. Journal of Computer-Mediated Communication, 16(4), 511-527.doi:10.1111/j.1083-6101.2011.0152 2.x

Zimet, G., Dahlem, N. W., Zimet, S. G., \& Farley, G. K. (1988). The multidimensional scale of perceived social support. Journal of Personality Assessment, 52, 30-41. doi: 10.1207/s15327752jpa5201_2

Zimet, G., Powell, S., Farley, G., Werkman, S., \& Berkoff, K. (1990). Psychometric characteristics of the multidimensional scale of perceived social support. Journal of Personality Assessment, 55(3), 610-617. doi: 2280326 